\title{
Mitochondrial genomes of two parasitic Cuscuta species lack clear evidence of horizontal gene transfer and retain unusually fragmented $\mathrm{ccmF}_{C}$ genes
}

\author{
Benjamin M. Anderson ${ }^{1 *} \mathbb{D}$, Kirsten Krause ${ }^{2}$ and Gitte Petersen ${ }^{1 *}$
}

\begin{abstract}
Background: The intimate association between parasitic plants and their hosts favours the exchange of genetic material, potentially leading to horizontal gene transfer (HGT) between plants. With the recent publication of several parasitic plant nuclear genomes, there has been considerable focus on such non-sexual exchange of genes. To enhance the picture on HGT events in a widely distributed parasitic genus, Cuscuta (dodders), we assembled and analyzed the organellar genomes of two recently sequenced species, C. australis and C. campestris, making this the first account of complete mitochondrial genomes (mitogenomes) for this genus.

Results: The mitogenomes are 265,696 and 275,898 bp in length and contain a typical set of mitochondrial genes, with 10 missing or pseudogenized genes often lost from angiosperm mitogenomes. Each mitogenome also possesses a structurally unusual $\mathrm{CCmF}_{C}$ gene, which exhibits splitting of one exon and a shift to trans-splicing of its intron. Based on phylogenetic analysis of mitochondrial genes from across angiosperms and similarity-based searches, there is little to no indication of HGT into the Cuscuta mitogenomes. A few candidate regions for plastome-to-mitogenome transfer were identified, with one suggestive of possible HGT.

Conclusions: The lack of HGT is surprising given examples from the nuclear genomes, and may be due in part to the relatively small size of the Cuscuta mitogenomes, limiting the capacity to integrate foreign sequences.
\end{abstract}

Keywords: Chloroplast, Dodder, Intracellular transfer, Mitogenome

\section{Background}

Horizontal gene transfer (HGT) may be broadly defined as the movement of genetic material between species by a means other than normal reproduction (e.g. fusion of gametes). The process has been inferred within and between both prokaryotes [1] and eukaryotes [2], and is now thought to potentially have a role in adaptation, as shown in recent examples from plants [3, 4]. While the

\footnotetext{
* Correspondence: banderson2914@gmail.com; gitte.petersen@su.se 'Department of Ecology, Environment and Plant Sciences, Stockholm University, SE-106 91 Stockholm, Sweden

Full list of author information is available at the end of the article
}

concept is sometimes extended to include transfers between cellular compartments (e.g. chloroplast to mitochondrion), we here refer to HGT as exchange of genetic material between organisms and consider it distinct from intracellular transfers.

Mechanisms that facilitate HGT between plants have been postulated to include direct contact via e.g. wounds/grafting [5], parasitic haustoria [6], epiphytic interactions [7] and illegitimate pollination [8]. Indirect routes have also been suggested, such as via soil, bacteria, viruses or endophytic fungi [8]. Early cases of detected HGT in plants $[9,10]$ were principally found in

(c) The Author(s). 2021 Open Access This article is licensed under a Creative Commons Attribution 4.0 International License, which permits use, sharing, adaptation, distribution and reproduction in any medium or format, as long as you give appropriate credit to the original author(s) and the source, provide a link to the Creative Commons licence, and indicate if changes were made. The images or other third party material in this article are included in the article's Creative Commons licence, unless indicated otherwise in a credit line to the material. If material is not included in the article's Creative Commons licence and your intended use is not permitted by statutory regulation or exceeds the permitted use, you will need to obtain permission directly from the copyright holder. To view a copy of this licence, visit http://creativecommons.org/licenses/by/4.0/ The Creative Commons Public Domain Dedication waiver (http://creativecommons.org/publicdomain/zero/1.0/) applies to the data made available in this article, unless otherwise stated in a credit line to the data. 
their mitochondrial genomes (mitogenomes), possibly partly as a result of the greater sequence availability at the time compared to the nuclear genome. Cellular mechanisms such as active DNA uptake [11] and organelle fusion and fission [12,13] have been referenced to explain why HGT is more prevalent in the mitogenome, at least compared with the extensively sequenced chloroplast genome (plastome). While examples of HGT from nuclear genomes are accumulating, mitogenome examples still remain a substantial portion of the evidence for HGT in plants.

A group of plants whose lifestyle makes them more likely to experience HGT are parasites [14, 15], largely because of their close physical interaction with their hosts via specialised connective organs called haustoria. Parasitic plants rely on other plants for water and often for a source of carbon, having some degree of photosynthetic ability (hemiparasites) or having lost it (holoparasites). The altered need for photosynthetic capacity has impacted the size and gene content of plastomes of many parasitic plants $[16,17]$. Parasites in the family Viscaceae also have markedly divergent and reduced gene content in their mitogenomes [18-20], but such a reduction was not observed in other groups of parasites $[20,21]$. The more common feature associated with parasitic plant mitochondria is the presence of foreign DNA. An extreme example of incorporated foreign DNA in a mitogenome is Lophophytum mirabile (Balanophoraceae), which is inferred to have acquired approximately $80 \%$ of its genes and $60 \%$ of its sequence from its host $[22,23]$. Another example includes members of Rafflesiaceae, for which up to $40 \%$ of their mitochondrial genes are inferred to have been transferred, with many of the putatively transferred genes shown to be actively transcribed [24], though whether the inferred transfers are indeed foreign and/or functional has recently been questioned [25].

Parasitic plants in the genus Cuscuta are twining stem parasites with reduced/absent leaves that rely on their host plants for both water and a carbon source obtained via haustorial connections [26]. Photosynthetic ability is variably retained in the genus, and there is a diversity of chloroplast structure and plastome gene content [2731]. For example, while some species are known to photosynthesize to some degree, carbon fixation in the bright yellow species $C$. campestris is below the compensation point [27] making it unable to survive and grow, let alone complete its life cycle, without its hosts. The range of possible host species for Cuscuta varies from narrow to especially broad with examples including members of Fabaceae, Apiaceae, Amaranthaceae, Asteraceae, Solanaceae, Linaceae, Ericaceae, Rutaceae and Amaryllidaceae among others [26]. The haustoria that Cuscuta plants use to obtain nutrients from their hosts are capable of both phloem and xylem connections [32] and are also capable of transmitting genetic material in the form of RNA [6] and DNA [4,33], which together with the phylogenetically broad host range makes Cuscuta a potentially ideal study system for exploring the phenomenon of HGT.

A number of studies have implicated Cuscuta species as sources and recipients of HGT events. Studies of host Plantago [34, 35] and Geranium [36] species show evidence of multiple mitochondrial genes likely obtained from Cuscuta parasites. Multiple transcriptomic surveys identified candidate nuclear genes in Cuscuta that may have been acquired by HGT [37-39]. Nuclear genome assemblies coupled with transcriptomic work have also revealed numerous candidates for HGT in Cuscuta campestris [4, 33]. A recent report of an Agrobacterium gene in three Cuscuta species could also represent an HGT from another plant, though it could have also been acquired directly from the bacterium [40]. To date the evidence suggests there is active exchange of genetic information between Cuscuta and its hosts, with donations of mitochondrial genes and acquisitions of multiple nuclear genes, but not yet any indication of imported mitochondrial genetic material.

While nuclear genomes for Cuscuta australis [41] and C. campestris [33] have been assembled, our knowledge of the mitogenome in Cuscuta is limited to a subset of genes from Cuscuta gronovii [36]. Given the lack of knowledge about Cuscuta mitogenomes and the expectation that the mitogenome is a prime location to look for HGT, we set out to assemble and characterise mitogenomes for Cuscuta australis and C. campestris. The complete genomes will enable us to assess the evidence for HGT, and secondarily intracellular transfers between genomic compartments, in Cuscuta mitogenomes, and test the unspoken assumption that examples of HGT in Cuscuta should be abundant.

\section{Results \\ Organelle genomes}

The plastomes were assembled as single circular molecules having typical quadripartite structure with two inverted repeats separating small and large single copy regions (see Figs. S1 and S2, Additional file 1). The plastomes are 85,263 and $86,749 \mathrm{bp}$, with GC contents of 37.8 and $37.7 \%$ for $C$. australis and C. campestris, respectively. Beyond the inverted repeats $(14,070$ and 14,348 bp in C. australis and C. campestris, respectively), there are no dispersed repeats longer than $50 \mathrm{bp}$ and with identity $>90 \%$. Tandem repeats make up approximately 0.2 and $0.4 \%$ of the plastomes of $C$. australis and C. campestris, respectively. Similar to other reduced plastomes of Cuscuta species such as C. gronovii [29] and C. obtusiflora [28], the plastomes are missing all 


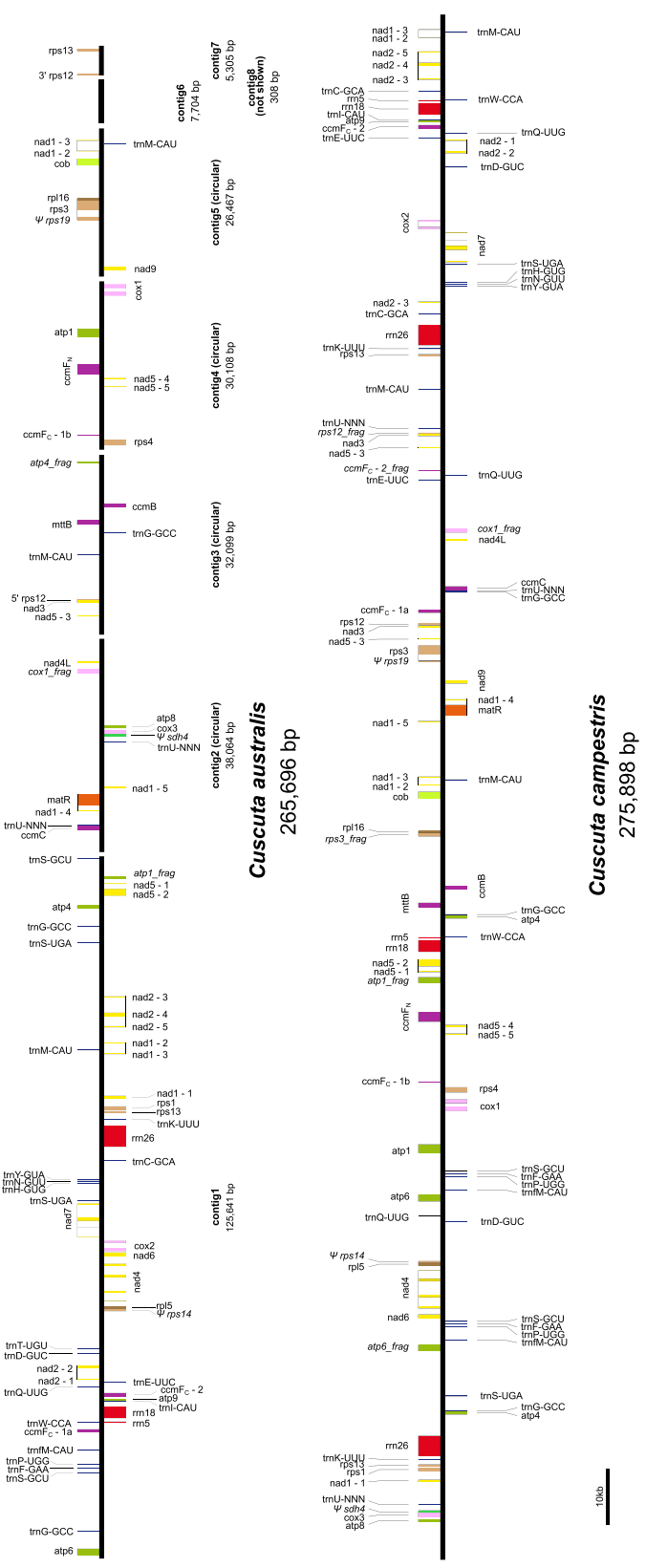

Fig. 1 Linear representations of mitogenomes of Cuscuta australis and C. campestris. The assembly of C. australis consists of eight contigs (divided by gaps), while that of $C$. campestris is represented as a single circle. Pseudogenes are indicated with a capital psi $(\Psi)$. Fragmented genes have "_frag" at the end of their labels. Transcription is to the right above the lines, and to the left below the lines

$n d h$ genes (a pseudogenized copy of $n d h B$ is present in C. campestris), infA, matK, psaI, all rpo genes, rpl23, rpl32, and rps16. Missing tRNAs include trnA-UGC, trnG-UCC, trnI-GAU, trnK-UUU, trnR-ACG, and trnVUAC.

The mitogenomes were assembled as 265,696 and $275,898 \mathrm{bp}$, with GC contents of 44.0 and $44.3 \%$ for $C$. australis and $C$. campestris, respectively (Fig. 1). Dispersed repeats (see Table 1; Fig. S3, Additional file 1) comprise approximately 21.8 and $34.1 \%$ while tandem repeats make up approximately 5.0 and $2.5 \%$ of the mitogenomes of $C$. australis and C. campestris, respectively. Read depth was largely consistent across the assemblies, except for drops at ends of contigs and in some locations possibly representing alternative conformations (see Fig. S4, Additional file 1). Recombinational activity as assessed with long repeats varied from 10 to $48 \%$ (average $=29 \%, n=6$ ) and $33-47 \%$ (average $=$ 
Table 1 Features of the mitogenomes of Cuscuta australis, C. campestris and their close relative Ipomoea nil (NC_031158)

\begin{tabular}{|c|c|c|c|}
\hline Feature & C. australis & C. campestris & Ipomoea nil \\
\hline Length (bp) & 265,696 & 275,898 & 265,768 \\
\hline GC content (\%) & 44.0 & 44.3 & 44.5 \\
\hline Dispersed repeats (kb) & 57.8 & 94.0 & 15.6 \\
\hline Tandem repeats (kb) & 13.2 & 6.89 & 0.67 \\
\hline \multicolumn{4}{|c|}{ Gene coding length / introns (bp) } \\
\hline atp1 & 1530 & 1530 & 1530 \\
\hline atp4 & 579 & 579 & 579 \\
\hline $\operatorname{atp} 6$ & 1173 & 1173 & 1155 \\
\hline $\operatorname{atp} 8$ & 477 & 477 & 477 \\
\hline $\operatorname{atp} 9$ & 285 & 285 & 258 \\
\hline $\mathrm{ccm} B$ & 627 & 627 & 627 \\
\hline $\mathrm{ccmC}$ & 726 & 726 & 747 \\
\hline $\mathrm{ccmF}_{\mathrm{C}_{-}} 1 \mathrm{a}$ & 501 & 501 & 531 ("pseudo") \\
\hline $\mathrm{ccmF}_{C_{-}} 1 \mathrm{~b}$ & 128 & 128 & 92 ("pseudo") \\
\hline $\mathrm{ccmF}_{\mathrm{C}_{-} 2} 2$ & 640 & 640 & 562 ("pseudo") \\
\hline$c c m F_{N}$ & 1746 & 1755 & 1764 \\
\hline$c o b$ & 1179 & 1179 & 1182 \\
\hline $\operatorname{cox} 1$ & 1584 / 509 & 1584 / 534 & 1584 / 971 \\
\hline $\cos 2$ & $1023 / 854$ & $774 / 867$ & $783 / 1343$ \\
\hline $\operatorname{cox} 3$ & 798 & 798 & 798 \\
\hline matR & 1911 & 1911 & 1968 \\
\hline$m t t B$ & 774 & 774 & 840 ("pseudo") \\
\hline nad1_1 & 386 & 386 & 385 \\
\hline nad1_2-3 & $274 / 1772$ & $274 / 1223$ & $275 / 1445$ \\
\hline nad1_4-5 & 318 / 3982 & $318 / 3776$ & $318 / 3604$ \\
\hline nad2_1-2 & $546 / 1941$ & $546 / 1870$ & $545 / 1233$ \\
\hline nad2_3-5 & $921 / 2785,1833$ & $921 / 2348,1773$ & 922 / 2417, 1455 \\
\hline nad3 & 357 & 357 & 357 \\
\hline nad4 & 1488 / 1478, 2359, 1398 & 1488 / 1498, 2390, 1426 & 1488 / 1418, 3200, 2827 \\
\hline nad4L & 303 & 303 & 300 \\
\hline nad5_1-2 & $1446 / 838$ & $1446 / 857$ & $1446 / 844$ \\
\hline nad5_3 & 22 & 22 & 22 \\
\hline nad5_4-5 & $542 / 1088$ & $542 / 1175$ & $542 / 1339$ \\
\hline nad6 & 618 & 618 & 618 \\
\hline nad7 & $1176 / 1553,1124,2144$ & $1176 / 1294,910,1957$ & 1185 / 912, 1468, 1951 \\
\hline nad9 & 573 & 573 & 573 \\
\hline$r p / 2$ & - & - & - \\
\hline rp/5 & 555 & 555 & 555 \\
\hline rp/10 & - & - & 489 \\
\hline$r p / 16$ & 516 & 456 & 516 \\
\hline rps1 & 612 & 612 & 597 \\
\hline rps2 & - & - & - \\
\hline rps3 & 1830 / 1057 & 1794 / 996 & $1671 / 1510$ \\
\hline rps4 & 912 & 909 & 933 \\
\hline
\end{tabular}


Table 1 Features of the mitogenomes of Cuscuta australis, C. campestris and their close relative Ipomoea nil (NC_031158) (Continued)

\begin{tabular}{|c|c|c|c|}
\hline Feature & C. australis & C. campestris & Ipomoea nil \\
\hline rps7 & - & - & - \\
\hline rpsio & - & - & 363 / 902 \\
\hline rps 11 & - & - & - \\
\hline rps 12 & 378 & 378 & 378 \\
\hline rps 13 & 351 & 351 & 351 \\
\hline rps 14 & 276 (pseudo) & 258 (pseudo) & 303 \\
\hline rps19 & 209 (pseudo) & 114 (pseudo) & 285 \\
\hline $\operatorname{sdh} 3$ & - & - & - \\
\hline sdh4 & 431 (pseudo) & 486 (pseudo) & 408 \\
\hline$r r n 5$ & 116 & 116 & 119 \\
\hline$r r n 18$ & 2075 & 2037 & 1943 \\
\hline $\operatorname{rrn} 26$ & 3753 & 3656 & 3377 \\
\hline $\operatorname{trnC}-G C A$ & 71 & 71 & 71 \\
\hline $\operatorname{trnD}-G U C$ & 74 & 74 & 74 \\
\hline $\operatorname{trnE-UUC}$ & 72 & 72 & 72 \\
\hline $\operatorname{trn} F-G A A$ & 74 & 74 & 74 \\
\hline $\operatorname{trnG-GCC}$ & 72 & 72 & 72 \\
\hline $\operatorname{trnH}-G \cup G$ & 74 & 74 & 74 \\
\hline trnl-CAU & 74 & 74 & 74 \\
\hline $\operatorname{trnK} K-U U U$ & 73 & 73 & 73 \\
\hline $\operatorname{trnM}-C A U-c p$ & 73 & 73 & 73 \\
\hline trnfM-CAU & 74 & 74 & 74,74 \\
\hline $\operatorname{trnN}-G \cup U$ & 72 & 72 & 72 \\
\hline $\operatorname{trn} P-U G G$ & 75 & 75 & 74 \\
\hline $\operatorname{trn} Q-U U G$ & 72 & 72 & 72 \\
\hline $\operatorname{trn} Q-U \cup G-c p$ & - & 72 & - \\
\hline $\operatorname{trnS}-G C U$ & 88 & 88 & 88 \\
\hline $\operatorname{trnS}-G G A$ & - & - & 88 \\
\hline $\operatorname{trnS}-U G A$ & 87 & 87 & 87 \\
\hline $\operatorname{trnT}$-UGU & 72 & - & - \\
\hline $\operatorname{trn} W-C C A$ & 74 & 74 & 74 \\
\hline $\operatorname{trn} Y-G \cup A$ & 83 & 83 & 83 \\
\hline
\end{tabular}

Table showing a summary of the three mitogenomes, including gene content. Protein-coding, ribosomal RNA and transfer RNA genes often found in angiosperm mitogenomes are listed along with their lengths, the lengths of their introns, and whether they are missing ("-") or pseudogenized ("pseudo")

$37 \%, n=12$ ) for $C$. australis and C. campestris, respectively (see Tables S1 and S2, Additional file 1). For each mitogenome, we annotated 31 proteincoding genes, 3 ribosomal RNA genes, and 19 tRNAs (some in multiple copies; Table 1). Compared to $C$. australis, C. campestris has an extra trnQ-UUG of plastome origin (within a possibly transferred region), but trnT-UGU is missing. Common missing or pseudogenized genes for both genomes include $s d h 3$ and $s d h 4, r p l 2$ and rpl10, and six rps genes (rps2, 7, $10,11,14,19)$. Some extra fragments of already present genes broken up by possible alternative paths through the assembly include atp1 and $\operatorname{cox} 1$ for both species, and atp6, rps12 and rps3 for $C$. campestris.

The $c c m F_{C}$ gene was problematic to annotate in both species (annotated as a pseudogene in C. gronovii and Ipomoea nil) and may not be properly transcribed, as it appears to be both trans-spliced and missing a portion of the first exon. Based on alignments with other angiosperms, including the intron-less Platycodon grandiflorus (NC_035958), it appears that the $c c m F_{C}$ gene is split across three locations in both of the Cuscuta mitogenomes (Fig. 2). A large portion of exon 1 is separated 


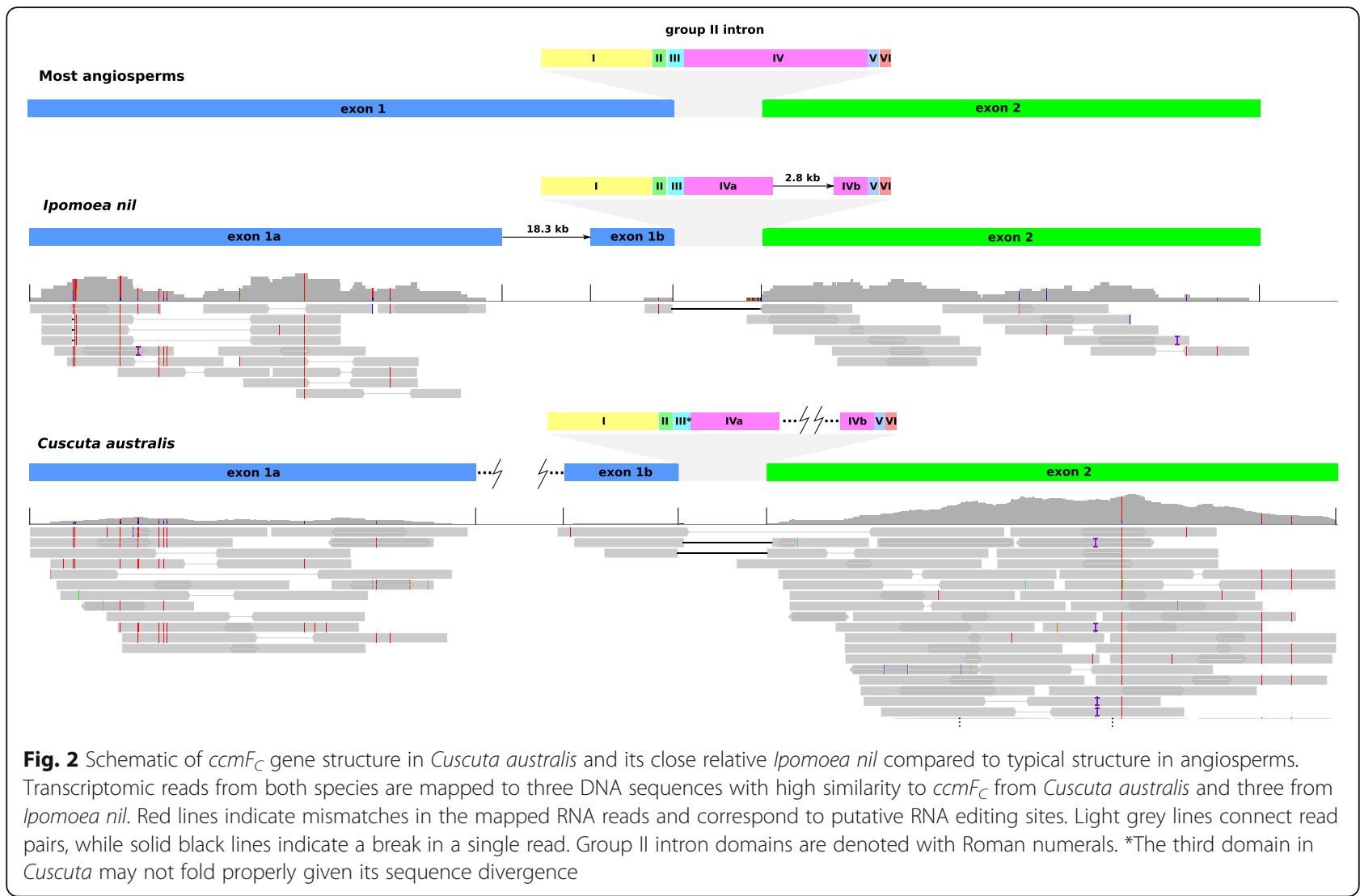

from the $3^{\prime}$ end of exon 1 and most of the group II intron. The remainder of the intron plus exon 2 are in a third location. Mapping mRNA reads resulted in low coverage of the $c c m F_{C}$ gene, but provided some support for successful trans-splicing of the $3^{\prime}$ end of exon 1 and exon 2 (see Fig. 2). There was no indication of an mRNA product that included both portions of exon 1, however, making it unclear whether the majority of exon 1 is actually translated into part of a functional product. PREPMT predicted 14 editing sites, three of which didn't have transcriptome support, and missed four sites that appeared to be RNA edited based on transcriptome read mapping (see Fig. S5, Additional file 1). Mapping mRNA reads against other mitochondrial gene sequences (e.g. atp6, $\mathrm{ccm}$; ; data not shown) recovered similarly low read depths as the mapping to $c c m F_{C}$, suggesting read depth in this case is unreliable for effectively determining relative expression of mitochondrial genes. For accurate investigation of mitochondrial transcription of the genes of interest, other techniques like mitochondrial run-on transcription assays [42] would be more suitable.

Another odd gene structure found in both Cuscuta species is the final portion of nad1, namely exon 4, $m a t R$ and exon 5 (see Fig. S6 Additional file 2). In the two Cuscuta assemblies, exon 5 is found in the reverse orientation on the opposite strand, while exon 4 is located on the same strand as matR. This may indicate nad1i728 has shifted to trans-splicing (as seen in some other angiosperms, e.g. Nicotiana tabacum, NC_006581.1), but if that is the case, it seems odd that exon 5 is in such close proximity to the rest of the intron and exon 4.

\section{Potential intracellular transfers}

Using reference plastomes, we plotted plastid hits on the mitogenome assemblies (see Figs. S7 and S8, Additional file 2). Excluding hits that occurred in the ribosomal genes or atp1, which are similar to plastid ribosomal genes and $\operatorname{atp} A$, we found 8 regions with hits, representing approximately 0.6 and $1.1 \%$ of the mitogenomes of C. australis and C. campestris, respectively (Table 2).

Phylogenetic trees for hits 2 and 7 (see Fig. S9, Additional file 2) support the mitochondrial sequences grouping with Cuscuta or Ipomoea chloroplast sequences, consistent with intracellular transfer. Both species share two regions corresponding to HMMER hits to plastid genes $n d h B$ and $p s b B$ (hits 4 and 5). Phylogenetic analysis of hits 4 and 5 are partly unresolved but suggestive of potential HGT, as the two Cuscuta species lack intact copies of $n d h B$ in their plastomes (hit 4) and 
Table 2 Reference plastome hits in the mitogenomes of Cuscuta australis and C. campestris

\begin{tabular}{|c|c|c|c|c|c|c|}
\hline Hit & Species & $\begin{array}{l}\text { Location: (contig) } \\
\text { coordinate }\end{array}$ & $\begin{array}{l}\text { Length } \\
\text { (bp) }\end{array}$ & $\begin{array}{l}\% \\
\text { identity }\end{array}$ & $\begin{array}{l}\text { Putative } \\
\text { origin }^{\mathrm{a}}\end{array}$ & Note \\
\hline 1 & $\begin{array}{l}\text { C. australis } \\
\text { C. } \\
\text { campestris }\end{array}$ & $\begin{array}{l}\text { (1) } 36,607 . .36,722 \\
60,410 . .60,295 \\
248,718 . .248,603\end{array}$ & 116 & $90-94$ & homology & trnD-GUC; identical to Ipomoea nil \\
\hline 2 & C. australis & (1) $84,001 . .84,148$ & 148 & $71-78$ & intracellular & $\begin{array}{l}\text { unresolved but close to Solanum, Ipomoea and Cuscuta } \\
\text { africana }\end{array}$ \\
\hline 3 & $\begin{array}{l}\text { C. australis } \\
\text { C. } \\
\text { campestris }\end{array}$ & $\begin{array}{l}\text { (1) } 119,219 . .119,360 \\
106,543 . .106,402\end{array}$ & 142 & $69-75$ & homology & portion of nad5 hitting portion of ndhF \\
\hline 4 & $\begin{array}{l}\text { C. australis } \\
\text { C. } \\
\text { campestris }\end{array}$ & $\begin{array}{l}\text { (2) } 16,249 . .15,698 \\
144,976 . .145,546\end{array}$ & $\begin{array}{l}552 \\
571\end{array}$ & $71-82$ & HGT? & portion of $n d h B$ and intron \\
\hline 5 & $\begin{array}{l}\text { C. australis } \\
\text { C. } \\
\text { campestris }\end{array}$ & $\begin{array}{l}\text { (2) } 17,873 . .17,432 \\
143,582 . .143,960\end{array}$ & $\begin{array}{l}442 \\
379\end{array}$ & $63-96$ & HGT? & portion of $p s b B$ \\
\hline 6 & $\begin{array}{l}\text { C. australis } \\
\text { C. } \\
\text { campestris }\end{array}$ & $\begin{array}{l}\text { (5) } 990 . .1101 \\
156,265 . .156,376\end{array}$ & 112 & $74-79$ & mitogenome & hits within ycf2; $100 \%$ identical to Solanales mitogenomes \\
\hline 7 & $\begin{array}{l}\text { C. } \\
\text { campestris }\end{array}$ & $61,212.62,827$ & 1616 & $82-96$ & intracellular & resolved closest to Cuscuta spp. \\
\hline 8 & $\begin{array}{l}\text { C. } \\
\text { campestris }\end{array}$ & $166,948 . .167,125$ & 178 & 73 & mitogenome & $\begin{array}{l}\text { hits unannotated; > 98\% identical to Solanales } \\
\text { mitogenomes }\end{array}$ \\
\hline
\end{tabular}

Table shows the locations and features of reference plastome hits in the mitogenomes of Cuscuta australis and C. campestris. Notes indicate further detail and phylogenetic placements or NCBI BLASTN results

${ }^{\text {a }}$ Putative origins are listed as explanations for the presence of the hit and are as follows: 1) homology: the hit likely arises from ancient similarity/transfer between plastomes and mitogenomes; 2 ) intracellular: the hit likely represents a transfer from the plastome to the mitogenome within the lineage; 3 ) HGT: the hit likely represents a transfer from another lineage, either plastome to mitogenome (intra) then mitogenome to mitogenome (HGT), or plastome to mitogenome (HGT); 4) mitogenome: the hit likely represents mitogenome sequence with some similarity to the plastome, possibly by chance, and not differing from related mitogenomes

the $p s b B$ hits group closer to Malpighiales than other Cuscuta chloroplast sequences. In both Cuscuta species, the $p s b B$ and $n d h B$ hit regions are within $2 \mathrm{~kb}$ of each other, possibly indicating a larger transferred region. Bootstrap support is low in the $n d h B$ tree, with taxa from asterids and rosids, making it unclear whether the two hits are associated with the same potential donor lineage.

Searches of the nuclear genomes produced markedly different results for the two Cuscuta species. In C. australis, the $\sim 260 \mathrm{Mbp}$ assembly hit the mitochondrial assembly for cumulatively $\sim 27 \%$ of its length, but in $C$. campestris, the $\sim 477 \mathrm{Mbp}$ assembly hit the mitochondrial assembly for $\sim 99.8 \%$ of its length or essentially the entire assembly. Three scaffolds with lengths of 172, 43 and $21 \mathrm{~kb}$ are essentially entirely mitochondrial. These scaffolds could tentatively indicate recent large-scale integration of mitochondrial sequences into the nuclear genome, but could also be simple assembly artifacts since mitochondrial contaminations are difficult to identify.

Table 3 Nuclear candidate copies of missing mitochondrial genes in Cuscuta australis and C. campestris

\begin{tabular}{llll}
\hline $\begin{array}{l}\text { Mitochondrial } \\
\text { gene }\end{array}$ & $\begin{array}{l}\text { Nuclear copy length } \\
\text { (bp) }\end{array}$ & $\begin{array}{l}\text { Reference average length } \\
(\mathbf{b p )}\end{array}$ & Putative origin \\
\hline rps2 & 618 & 978 & $\begin{array}{l}\text { vertical; nuclear copy or UTP--glucose-1-phosphate } \\
\text { uridylyltransferase }\end{array}$ \\
rps10 & 387 & 354 & unclear; possible HGT \\
rps11 & 486 & vertical; nuclear copy \\
rps14 & 432 & 295 & vertical; nuclear copy \\
rps19 & 321 & 286 & unclear; possible HGT \\
sdh3 & 273 & 343 & vertical; nuclear copy \\
sdh4 & 321,324 & 418 & unclear; possible HGT \\
\hline
\end{tabular}

Table showing the nuclear candidate copies, including the lengths of reference mitochondrial genes. The putative origin is based on phylogenetic analysis of top NCBI BLASTN hits together with reference mitogenomes 


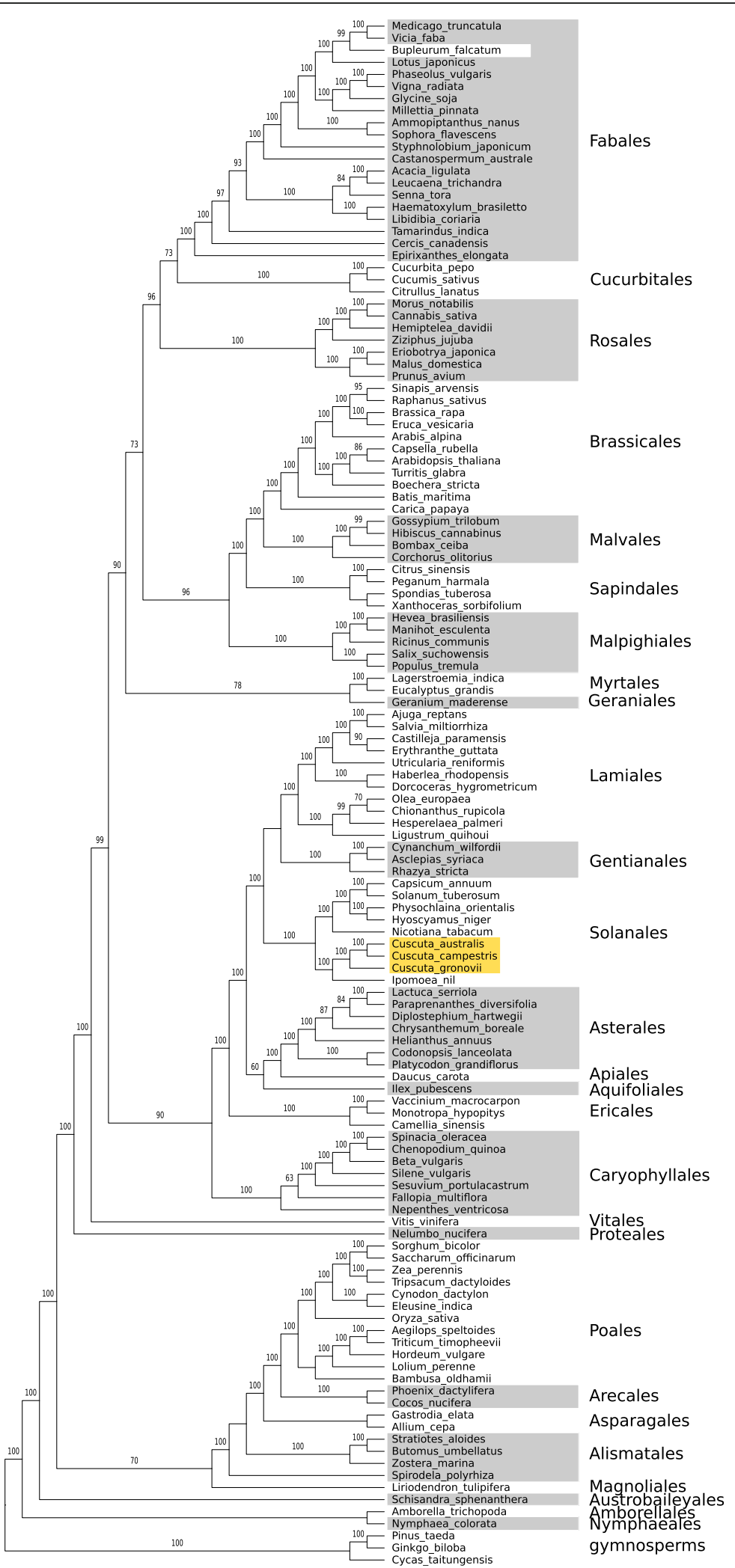

Fig. 3 (See legend on next page.) 
(See figure on previous page.)

Fig. 3 Maximum likelihood cladogram based on concatenation of all known mitochondrial genes present in Cuscuta together with

representatives from angiosperm orders with fully assembled mitogenomes. Orders are indicated at the right along with the outgroup

gymnosperms, and the location of Cuscuta is highlighted in yellow. Bootstrap support of $60 \%$ or greater is shown above branches. The sequence

for Bupleurum falcatum (NC_035962.1) is likely misidentified

Potential candidates for missing mitochondrial genes were identified in both nuclear genomes for rps2, rps10, rps11, rps14, rps19, sdh3 and sdh4 (Table 3). All the candidates had intact open reading frames except for $s d h 3$, which had no determined start codon. Phylogenetic analysis of the candidates with copies from the reference mitogenomes and top NCBI BLASTN hits consistently put the nuclear copies on longer branches relative to the mitogenome copies (see Figs. S10-13, Additional file 2). In some cases, the trees suggest vertical inheritance of a nuclear copy (rps2, rps11, rps14, sdh3) but in three cases it is unclear and could represent HGT (rps10, rps19, $s d h 4)$, though long branch attraction may obscure phylogenetic relationships. Searching for indications of mitochondrial targeting sequences with Predotar v. 1.04 [43] indicated possible mitochondrial targeting ( $\mathrm{p}$ 0.21-0.44) for rps10, rps14, rps19 and $s d h 3$ (if an ' $\mathrm{M}$ ' is added in place of the undetermined start codon).

\section{Horizontal gene transfer}

Phylogenetic analysis of the concatenation of all mitochondrial genes strongly supports the grouping of Cuscuta within Solanales (Fig. 3). Based on the individual gene trees with sampling across seed plants, the majority of genes $(26 / 34,76 \%)$ in the three Cuscuta species are strongly supported ( $>80 \%$ bootstrap support) as grouping together and sister to their close autotrophic relative Ipomoea nil, a pattern consistent with vertical rather than horizontal inheritance (see Fig. S14, Additional file 3). Exceptions to this pattern are atp 9 (71\% sister to Ipomoea), $\operatorname{cmB}$ (unsupported), cox2 (66\%), nad3 (unsupported), nad4L (not sister to Ipomoea, but $80 \%$ support together with Ipomoea in Solanales), rpl16 (68\%), rps12 (unsupported), and rrn5 (unresolved) (note that $c c m F_{C}$ and $r r n 5$ from $C$. gronovii were not included). In the cases with no support for a sister relationship with Ipomoea, there is no strong support for a grouping elsewhere, as would be expected for HGT.

Intergenic sequences generally lacked clear evidence of longer (> $500 \mathrm{bp}$ ) contiguous groups of hits to single orders outside Solanales (see Figs. S7 and S8, Additional file 2). Some exceptions could be identified, including hits to Asterales, Caryophyllales, Fabales, Gentianales, Lamiales and Vitales, totalling approximately 7.4 and $4.7 \%$ of the mitogenomes of C. australis and C. campestris, respectively.

In portions of some genes, blast hits with higher identity to other orders than to Solanales were found ( $\operatorname{cox} 2$ exon 1, nad4 exon 3, and nad5 exon 5), so we ran GENECONV to detect possible conversion. In two cases there were some sequences showing significant $(P<$ 0.05 ) signal of conversion (4 sequences for cox 2 and 2 for nad5), but not involving the Cuscuta sequences. For nad4, there were many significant comparisons, but all but one involved Vicia faba (KC189947), suggesting it may have undergone gene conversion. There was no significant indication that the Cuscuta sequences were chimeric.

\section{Discussion}

Recently sequenced genomes of parasitic Cuscuta species revealed extensive HGT events into their nuclear genomes from a broad range of non-parasitic angiosperms that serve as their hosts. It was a reasonable expectation that there would be high transfer activity of genetic material into the mitogenomes of these parasites as well. The organellar genomes of the two Cuscuta species that we have assembled and annotated here, however, are not clearly different in gene content from other Cuscuta plastomes or typical angiosperm mitogenomes. Based on phylogenetic analysis, we found little evidence for HGT into the mitogenomes of the two species, an unexpected result given the parasitic lifestyle of Cuscuta and evidence for HGT candidates in their nuclear genomes. In the process of examining the mitogenomes, we also identified a novel structure of the $c c m F_{C}$ gene in Ipomoea and Cuscuta, indicative of a shift to transsplicing of the group II intron and possibly a shorter product.

\section{Gene losses and possible intracellular transfers}

All of the 10 genes missing from the two Cuscuta mitogenomes are commonly found to be lost during angiosperm evolution [44, 45]. That rps2 and rps11 are missing is consistent with the inference of their ancient losses in angiosperms [44]. For 7 of the 10 missing or pseudogenized genes, potential candidates for nuclear copies were found. Transfers of mitochondrial genes to the nucleus have been inferred to be numerous across plant evolution and seem to be the primary mode of functional gene loss from the mitogenome [46]. Whether the gene products from the nuclear candidates are functional or imported into the mitochondrion is uncertain, but tests for targeting sequences suggested some of them might be targeted to the mitochondrion. The nuclear candidates are on longer branches in phylogenetic trees 
compared to the mitochondrial copies, suggesting higher substitution rates. This is consistent with typical observations of higher substitution rates in the nuclear genome compared to the mitogenome [47], and of higher rates in nuclear copies of genes transferred from the mitogenome compared to the mitogenome copies [48], though exceptions exist, such as in Silene [49].

The three missing mitochondrial genes not detected in the nuclear genomes are $r p l 2, r p l 10$ and rps7. Losses of rpl2 and rps7 are frequent among angiosperms, but only for $r p l 2$ are losses commonly associated with functional transfers to the nucleus [36, 44, 50, 51]. Losses of rpl10 are less common and have been associated with functional replacement by a duplicated nuclear gene normally encoding chloroplast RPL10 in some monocot and Brassicaceae lineages $[45,52,53]$. Searching ORFs from both nuclear genomes for possible copies of RPL10 suggested the presence of one ( $C$. australis) or two ( $C$. campestris) genes more similar to the chloroplast-targeted copy from Arabidopsis (NP_196855) than to the mitochondrial-targeted one (NP_187843). Targeting predictions from Predotar for the copies in Cuscuta provided no support for targeting the chloroplast or the mitochondrion.

Plastid transfers appear to be limited in the mitogenomes, with a $148 \mathrm{bp}$ hit in C. australis and a $1.6 \mathrm{~kb}$ hit in C. campestris. The other main candidate regions included hits to $p s b B$ and $n d h B$, but based on phylogenetic analysis appear to be more similar to regions in distantly related plants. The trees are inconclusive as to the origin of the region, but suggest a horizontal transfer, possibly via an intracellular plastome transfer to the donor's mitogenome and then a plant to plant transfer between mitogenomes, a common route for plastome to mitogenome HGT [54]. The close association of the $p s b B$ and $n d h B$ hits might indicate a transfer of a larger contiguous block of DNA from a donor with the genes in close proximity, but this is speculative and the donor lineage is not clear from our search and trees.

\section{Lack of horizontal gene transfer}

Phylogenetic evidence for HGT in the Cuscuta mitogenome assemblies is lacking, with only a few genes having $<80 \%$ bootstrap support for a close relationship to Ipomoea nil, and those exceptions also with negligible support for alternative relationships. BLASTN-based assessment of intergenic sequences did uncover a few longer candidates for possible HGT, but it is unclear how reliable the similarity search is for uncovering the origin of those sequences. It is possible they represent vertically-inherited mitogenomic sequences. Given the dynamic nature of mitogenome size and structure in angiosperms [49, 55-57], our limited sampling of lineages may not include more similar sequences from more closely related plants. The HGT candidates also lack embedded genes for the most part, making it difficult to determine homology and to further support an origin. In cases where BLAST hits to another order cover genes (cox3, nad4L, rps13), phylogenetic analysis of the genes generally contradicts the BLAST-based assignment. The aforementioned presence of sequences of chloroplast origin similar to regions in distantly related plants may also indicate that some HGT has occurred, possibly earlier in the evolution of Cuscuta given the region is shared by all the Cuscuta sequences we examined.

Why the mitogenomes lack clear evidence of HGT is unclear, given it is prevalent in the nuclear genome of Cuscuta. One possible explanation may be related to the size of the mitogenomes we have assembled, which are on the low side for angiosperms. Based on the reference set of mitogenomes (with 168 angiosperms), the average size of the angiosperm mitogenomes is roughly $670 \mathrm{~kb}$, while the assemblies are less than half that. Given there has been a correspondence observed between mitogenome size and the amount of incorporated plastid transfers [54] and that plant mitochondrial genomes appear to have relaxed restriction on the size of intergenic spacers (e.g. [58]), it may be that larger mitogenomes with longer intergenic regions allow for the incorporation of more foreign DNA. Conversely, the smaller mitogenomes in Cuscuta may experience more restriction on what can be successfully incorporated. Other explanations could include population sizes and demographic effects that make it more or less likely to retain incorporations of foreign DNA in a species.

It is unclear why there has not been an extensive expansion of intergenic material in Cuscuta or Ipomoea for that matter $(265,768 \mathrm{bp})$. The wide range of sizes of angiosperm mitogenomes are difficult to explain, but recombination rates have been put forward as one potential factor, albeit a complex one [49]. In a study of the massive differences in size amongst Silene mitogenomes, Sloan et al. [49] found that the larger mitogenomes experienced lower recombinational activity $(<20 \%)$ and had more divergent repeats, suggesting reduced gene conversion. The assembled mitogenomes had relatively high recombinational activity across longer repeat sequences as assessed with long reads (often $>20$ and $>$ $40 \%$ for C. australis and C. campestris, respectively), though with small sample sizes. The difficulty in assembling the mitogenomes and the multiple potential paths through the assemblies are also consistent with multiple conformations across highly similar or identical repeats, possibly indicating ongoing recombination and conversion.

Curious structure of $\mathrm{ccmF}_{\mathrm{C}}$ and its trans-splicing intron Both Cuscuta species and their close autotrophic relative Ipomoea nil appear to share a different structure of their 
$c c m F_{C}$ genes than other plants. In all three cases, the first exon is split into two pieces, and the same has happened for the group II intron, making trans-splicing of the intron necessary in order to join the now remotely located parts of the gene. In angiosperm mitogenomes, most introns are group II introns (having six domains) and require multiple protein factors, including some encoded in the nuclear genome, for proper splicing [59]. While the exact mechanism of trans-splicing for group II introns and the splicing factors required remain unclear, this type of splicing has been observed in a number of other angiosperm mitochondrial genes, namely nad1, nad2 and nad5 [60] and cox2 [61]. Typically, the break point for the two pieces of the intron that are trans-spliced is in domain IV $[60,62]$, which is where the intron is broken in Cuscuta and Ipomoea. The evidence presented here, namely the mRNA reads that indicate a product joined from two disparate locations in the genome and the break in domain IV, suggests this is the fifth such example of a gene with a group II intron that has shifted to trans-splicing.

Angiosperm $c c m F_{C}$ genes examined so far typically have intact rather than broken group II introns. For splicing of the intact intron, an essential nuclear-encoded splicing factor, WTF9, has been identified which shares an RNA-binding domain with another factor involved in intron splicing in plastids [63]. In examining the role of WTF9, des Francs-Small et al. [63] also elucidated the role of $c \mathrm{cmF} F_{C}$ in cytochrome biogenesis, showing that proper splicing of the intron for the formation of a complete protein was essential to the generation of cytochromes $\mathrm{c}$ and $\mathrm{c}_{1}$ in Arabidopsis. In addition, splicingimpaired mutants had higher levels of alternative oxidase, suggesting problems with the respiratory chain [63]. It appears that being able to produce a fully intact $c c m F_{C}$ product is important for respiratory function, which makes the break in the first exon in Cuscuta and Ipomoea puzzling. While there is evidence that the intron is trans-spliced and connects the $3^{\prime}$ portion of exon 1 with exon 2 , there is no indication that the larger $5^{\prime}$ portion of exon 1 is connected for translation into the final product. Transcriptomic reads show RNA editing in all parts of the gene (see Fig. 2 and Fig. S4, Additional file 1), similar to predictions from PREP-MT, but whether this indicates an intact product is unclear. Pseudogenization of $\mathrm{cmmF} F_{C}$ has only been reported for Silene conica amongst angiosperms [49], though there was indication that a truncated form of the protein might still be expressed and functional. Normally, the full $c \mathrm{~cm} F_{C}$ product is thought to be integrated into a larger complex potentially involved in heme ligation [64]. Another possible explanation for the process might be a continued trend of gene breakup, similar to the fission of $c c m F_{N}$ in Trifolium, Brassicaceae and Allium [65]. The functionality of at least part of the $c c m F_{C}$ gene would be consistent with its conservation in both Ipomoea and Cuscuta, though their copies are divergent relative to other members of Solanales (see Fig. S14, Additional file 3). Gene loci coding for homologs of the WTF9 splicing factor are present in C. campestris and C. australis (data not shown), excluding this as a reason for the switch. How Cuscuta and Ipomoea are able to function effectively with their $c c m F_{C}$ structure is unclear, and additional work would be helpful to characterise and evaluate the $c c m F_{C}$ protein products in this group of plants.

While the assembly approach is not guaranteed to have captured the entirety of the mitogenomes (for instance, the Cuscuta australis assembly consists of multiple contigs, and the C. campestris assembly was circularized from multiple smaller circular contigs), the search approach makes it unlikely that any missing sequences would carry substantially different phylogenetic signals of HGT than the bulk of the assemblies or that they would harbour intact foreign genes or a contiguous copy of $c c m F_{C}$. Alternative structural arrangement of the contigs and construction of the assemblies are also unlikely to affect our conclusions on HGT, but could have some impact on how we have interpreted the structure of $c c m F_{C}$. The lack of mRNA support for a fully intact $c c m F_{C}$ product, however, supports the assembly and interpretation of a fragmented gene structure.

\section{Conclusions}

Our search of the assembled mitogenomes of Cuscuta australis and C. campestris revealed little to no indication of HGT, contrary to our expectations based on examples from their nuclear genomes. This finding suggests that elevated rates of HGT into mitogenomes, where the bulk of examples from parasitic plants have been found to date, is not always the case. The observed amount of HGT may also be influenced by speciesspecific factors such as mitogenome size and recombination frequency that allow for any DNA uptake to be retained.

\section{Methods}

We downloaded from GenBank portions of previously published datasets from whole genome sequencing studies of Cuscuta australis and C. campestris [33, 41]. For C. australis, we downloaded $150 \mathrm{bp}$ paired-end reads from an Illumina $350 \mathrm{bp}$ insert library (SRA accession SRR5851367; $155 \mathrm{M}$ reads) and PacBio long reads (SRA accessions SRR5851361-6; $711 \mathrm{k}$ reads). For C. campestris, we downloaded 301 bp paired-end reads from Illumina $800 \mathrm{bp}$ insert libraries (SRA accessions ERR1917169-71; 9.5 $\mathrm{M}$ reads) and PacBio long reads (SRA accessions ERR1910865-7; $490 \mathrm{k}$ reads). Illumina 
short reads were evaluated with FastQC [66] before and after read deduplication, adapter and low quality base trimming, error correction and read merger using Clumpify, BBDuk, BBMerge and Tadpole in the BBMap package v. 38.08 ( [67]; https://sourceforge.net/projects/ bbmap/). As part of initial assembly, long reads were corrected then trimmed using default settings in Canu v. 1.8 [68]. After initial quality control, the datasets consisted of $1 \mathrm{M}$ merged and $122 \mathrm{M}$ unmerged short reads and $399 \mathrm{k}$ long reads for $C$. australis, and $316 \mathrm{k}$ merged and $7 \mathrm{M}$ unmerged short reads and $79 \mathrm{k}$ long reads for C. campestris.

For transcriptome data of C. australis [41], we downloaded $150 \mathrm{bp}$ paired-end reads for various tissues (SRR6664647-54; 411 M reads). After read deduplication and adapter and low quality base trimming using Clumpify and BBDuk, $268 \mathrm{M}$ reads were retained with an average read length of $142 \mathrm{bp}$ (72\% of reads were untrimmed). Transcriptomic reads for Ipomoea nil were obtained from three sources: the $1 \mathrm{KP}$ project [69], ERR2040616; a transcriptome study [70], SRR1772255; and a genome project [71], DRR024544-9. Reads were also deduplicated and trimmed for adapters and low quality bases, with $157 \mathrm{M}$ reads retained with an average read length of $97 \mathrm{bp}$ ( $81 \%$ of reads were untrimmed).

\section{Organelle genome assemblies}

We approached organellar assembly by running separate short and long read de novo assemblies, searching resulting contigs using BLASTN v. 2.6.0+ [72-74] with default parameters for hits to reference genomes (see Tables S3 and S4, Additional file 4), then using those contigs with hits as targets for further mapping with BBMap and assembly.

Short read datasets were first filtered using the Kmer Analysis Toolkit (KAT) v. 2.4.2 [75] to select a high coverage subset of reads for easier assembly. Coverage targets were based on mapping reads to sequences from reference plastome (Cuscuta exaltata, NC_009963) and mitogenome (Ipomoea nil, NC_031158) CDS. Based on the mapping, we chose kmer depth filters of 500-25,000 selecting $\sim 35 \mathrm{M}$ reads and $30-2100$ selecting $\sim 700 \mathrm{k}$ reads for $C$. australis and C. campestris, respectively. The filtered read sets were assembled using Unicycler v. 0.4.8 [76], which when given only short reads optimizes a SPAdes v. 3.14.0 [77] assembly.

Long read datasets were assembled in Canu v. 1.8 [68] using default settings and with genome size estimates of $280 \mathrm{M}$ and $550 \mathrm{M}$ for $C$. australis and C. campestris, respectively. In order to complete one run, we set the stopOnLowCoverage parameter to 0.1 for C. campestris.

We examined contigs from the short and long read assemblies in Bandage v. 0.8.1 [78] to identify those with BLAST (blastn megablast default parameters) hits to the reference genomes and which had similar depths (for distinguishing plastid and mitochondrial contigs). We considered as candidates contigs with $>50 \%$ coverage of hits to the respective references, and in cases of less than $50 \%$ coverage, whether read depth was similar to depth of contigs having greater coverage of hits. Contigs with hits and identified as putatively plastid or mitochondrial were used as targets for mapping the full sets of short reads to create input datasets for Unicycler. The mapped reads were assembled along with long reads (corrected and trimmed with Canu) in Unicycler, which assembles long reads with miniasm [79] and Racon v. 1.4.3 [80] and uses the assembly to resolve repeats and create bridges in the SPAdes small read assembly graph.

Plastome assemblies were obtained after a single round of Unicycler, producing single circular contigs. In order to complement the assembly approach, we also used NOVOPlasty v. 3.7.2 [81] with the deduplicated and adapter-trimmed (but not error corrected or merged) short read datasets. We used an $r b c L$ sequence from Cuscuta exaltata as a seed, which NOVOPlasty uses to select an initial matching read and begin iterative extension until it can circularize an assembly. The resulting two options per assembly (different configurations across the inverted repeats) were then compared with BLAST to the assemblies from Unicycler to verify the sequences were identical.

The mitogenome assemblies were more challenging, given the presence of long repeats and linear and circular contigs that in some cases could be joined at multiple locations, but this differed between assemblies. For $C$. australis, a second round of Unicycler was run after extending contigs by up to $100 \mathrm{kbp}$ using Tadpole (part of the BBMap package) with settings el $=100,000$ er $=$ 100,000 mode $=$ extend $k=31$ and the full short read dataset, and then mapping short reads again. Simplifying the assembly graph included removing small contigs that were fully present in a larger contig or were too short (e.g. < $100 \mathrm{bp}$ ). We then checked long read contigs from the initial assembly in Bandage to ensure they were covered by hits from the remaining assembly. Since there were four linear and four circular contigs, and since the circular contigs did not share repeats that could be used to merge them, we were unable to merge all contigs into a single contig. Repeats are present (though there are no long repeats shared between circles) and there are alternative paths through the assembly, so our representation is one of multiple possible and does not reflect the actual structure of the mitochondrial genome in the plants. While we were not able to merge the contigs in C. australis, we could join circular contigs for the assembly of C. campestris. For C. campestris, the first round of Unicycler produced four circular contigs along with three small linear contigs (linear totalling $<5 \mathrm{~kb}$ ) which hit the 
plastome or had low depth and were absent from the long read contigs. The short linear contigs were not included in further assembly. The four circles had shared long repeats, and these were examined using long reads to determine if multiple configurations were present, suggesting the separate contigs are sometimes contiguous. Long reads were mapped and aligned to extracted repeats with Minimap2 v. 2.14-r883 [79], Samtools v. 1.7 [82] and MAFFT v. 7.310 [83] and using a custom Python script including 'pysam' (https://github.com/ pysam-developers/pysam). When the reads indicated multiple configurations across the repeat, we manually joined the circles. The circles all shared repeats that showed multiple configurations, so we were able to merge the four circles into a single circular contig.

\section{Read mapping and annotation}

In order to check the mitogenome assemblies for clear errors, we conservatively mapped short and long reads then manually compared the resulting BAM files in IGV v. 2.6+ [84]. Short reads were mapped using BBMap with settings pairedonly $=\mathrm{t}$ minid $=0.9$ ambiguous $=$ random maxindel $=100$ strictmaxindel $=\mathrm{t}$ pairlen $=1000$. Long reads were mapped using Minimap2 in mode 'map-pb' and filtered with Samtools with option -F 2308, followed by filtering the resulting BAM file with a custom Python script using 'pysam' to only retain mappings where reads covered the target for at least $80 \%$ of their length. Long reads were used to check structural assembly and cases of insertions, where high coverage plastid reads can lead to misassembly of the mitogenomic sequence. The long reads passing through an insertion can be used to identify the lower-coverage short reads that are mitochondrial and allow for manual correction. For C. australis, only one location suggested a small (28 bp) sequence that needed to be deleted (absent in most long reads). For C. campestris, one location of a plastome insert needed three single base pair corrections and a small (9 bp) deletion to match the long reads that spanned the region rather than the higher depth plastid reads.

Plastomes and mitogenomes were annotated using custom scripts to BLAST (BLASTN, BLASTX, default search settings) against nucleotide and protein databases built using reference plastid (14 from 13 genera and 13 families, see Table S3, Additional file 4) and mitochondrial (172 from 130 genera and 62 families, see Table S4, Additional file 4) genomes, an approach similar to that used in Mitofy [85]. For mitogenomes, we also used the references to build protein alignments for constructing a HMMER v. 3.3 ( [86]; http://hmmer.org) database that we used to search open reading frames (ORFs) found using ORFfinder v. 0.4.3 (NCBI; https://www.ncbi.nlm. nih.gov/orffinder/). Following searches, hits were examined and adjusted manually, with input from MFAnnot v. 1.35 (https://github.com/BFL-lab/Mfannot) to select intron boundaries. Transfer RNAs were also detected and checked with tRNAscan-SE 2.0 [87]. Pseudogenes were subjectively annotated if hits had clear frameshifts and/or internal stop codons, and gene copies broken by repeats or alternative paths in the assembly were annotated as fragments.

Dispersed repeats were detected by searching the organellar genomes against themselves using BLASTN with an $E$ value of $1 \mathrm{e}-10$ and filtering out hits less than $50 \mathrm{bp}$ and lower than $90 \%$ identity. Overlapping hits were then filtered with a custom python script, and the percentage repeat content calculated. For visualization, the genomes were searched against themselves using Circoletto [88] with an $\mathrm{E}$ value chosen to approximate a $90 \%$ identity threshold (1e-70 and 1e-40 for C. australis and C. campestris, respectively). As a proxy for recombinational activity, we used long repeats (>500 bp, >90\% identity) and mapped long reads. A random selection of up to 50 long reads that mapped to each repeat were aligned with the repeat and evaluated manually for whether they showed indications of incongruence on either side of the repeat. The proportion of the mapped reads showing incongruence was used as a proxy for recombinational activity. Tandem repeat content was determined using Tandem Repeat Finder v. 4.09 [89] with recommended settings: 2778010 50,500. Organelle genome figures were based on output from OGDRAW [90].

To investigate the transcription and splicing of the mitochondrial $c c m F_{C}$ gene, we mapped mRNA reads using BBMap with the setting pairedonly $=\mathrm{t}$ against three DNA sequences manually stitched together and separated by N's from the annotated $c c m F_{C}$ locations in the $C$. australis assembly. We also aligned $c c m F_{C}$ DNA sequences from both Cuscuta species with $c c m F_{C}$ genes and CDS from reference mitogenomes using MAFFT to determine the locations of the exon and intron boundaries. We visualized alignments and mapping in Jalview $\mathrm{v}$. 2.11.0 [91] and IGV v. 2.8.0 [84]. Intron domains were roughly determined by iteratively running the Mfold web server [92] using the ccmFci829 from Nicotiana tabacum (NC_006581). RNA editing sites were predicted with PREP-MT [93] to compare with those observed in the transcriptomic reads. We also aligned Ipomoea nil transcriptomic sequences to similar $c c m F_{C}$ DNA sequences from its mitogenome to compare with Cuscuta.

To evaluate the notable gene structure of the final portion of nad1, we aligned sequences from Ipomoea nil, $C$. australis and C. campestris using Mauve v. snapshot 2015-02-13 [94]. We also mapped transcriptomic reads from $C$. australis to nad1 exons separated by $100 \mathrm{Ns}$ to evaluate splicing and whether there was evidence for an intact product. 


\section{Potential intracellular transfers}

To detect the presence of transfers from a plastome to the mitogenome, we searched each mitogenome assembly against the reference plastomes (Table S3, Additional file 4) using BLASTN with default parameters. We also searched the mitochondrial ORFs against a HMMER database built with the reference plastomes to detect whether there were intact portions of plastid genes present and to interpret blast hits. Broader searches used the NCBI non-redundant nucleotide database (https:// blast.ncbi.nlm.nih.gov/). We evaluated plastome hits of interest phylogenetically using top NCBI results in RAxML v. 8.2.12 [95] with rapid bootstrapping and a search for best tree in a single run, opting for 1000 bootstrap replicates under the GTRGAMMA model.

To determine whether any missing mitochondrial genes were present in the nuclear genome, and whether there were other transfers between the mitogenome and the nuclear genome, we conducted BLAST searches of the assemblies and of the reference mitogenomes (Table S4, Additional file 4) against the two published nuclear genomes (C. australis: GCA_003260385.1, C. campestris: PRJEB19879). For assessment of total hit coverage of the assemblies, we ignored hits $<250 \mathrm{bp}$ and with $<80 \%$ identity. For searches for missing genes, we ignored hits with $<50 \%$ identity.

\section{Horizontal gene transfer}

We investigated HGT using a phylogenetic approach, in which we built phylogenetic trees for each mitochondrial gene present in Cuscuta (as well as one based on all genes concatenated) and examined whether Cuscuta sequences were supported as grouping with their close relatives (particularly Ipomoea nil) or with more distantly related plants. Using reference mitogenomes (see Table S4, Additional file 4) and the Cuscuta assemblies along with data from C. gronovii (GenBank accessions KP940494-514), we extracted all CDS protein translations and corresponding nucleotide sequences, as well as nucleotide sequences for ribosomal RNAs. We removed some sequences in cases of high divergence (e.g. for Viscum scurruloideum) and when alignments suggested problematic annotations, and in some cases we had to reverse complement ribosomal RNA sequences. For each gene, we created protein alignments with ClustalOmega v. 1.2.4 [96] using default settings. We then converted the protein alignments back to nucleotide alignments with PAL2NAL v. 14 [97] using default settings. Alignments were run through PREP-MT [93] to predict editing sites, with sites having a prediction score of $>=0.5$ removed prior to analysis. Alignments were checked manually for apparent misalignments and problems with annotation around intron boundaries, and the coxl coconversion tract [98] was removed. For ribosomal RNA sequences, we aligned with MAFFT v. 7.310 using options --localpair and --maxiterate 1000. For each alignment we used a custom python script to eliminate positions with $>50 \%$ missing data, then ran RAxML v. 8.2.12 [95] with rapid bootstrapping and a search for best tree in a single run, opting for 1000 bootstrap replicates under the GTRGAMMA model. Finally, we plotted the resulting trees using $\mathrm{R}$ package ape [99], rooting them on non-angiosperm seed plants, and manually examined whether Cuscuta sequences grouped together, with their close relative Ipomoea nil, and with Solanales or whether they grouped elsewhere with bootstrap support $>80 \%$.

To explore intergenic sequences, we searched the reference mitogenomes against the Cuscuta assemblies using BLASTN with default parameters, removing hits < $250 \mathrm{bp}$ and with $<80 \%$ identity. For clearer display, we used custom scripts to filter BLAST hits so that hits of equivalent length (within $50 \mathrm{bp}$ ) or less and more than $2 \%$ lower identity than top hits were removed along the assembly. We plotted hits using the R package Sushi v. 1.26.0 [100]. We then manually examined hits in intergenic regions, looking for where the top hits were not from Solanales and evaluating whether the hits were suggestive of longer, potentially transferred tracts.

In cases of possibly chimeric gene sequences based on top blast hits, we assessed evidence for conversion using GENECONV v. 1.81 [101].

\section{Abbreviations}

CDS: Coding sequence; HGT: Horizontal gene transfer; ORF: Open reading frame.

\section{Supplementary Information}

The online version contains supplementary material available at https://doi. org/10.1186/s12864-021-08105-z.

Additional file 1: Fig. S1. Chloroplast genome of Cuscuta australis. Transcription is counterclockwise around the outside of the circle and clockwise inside. The grey inner graph is GC content. Abbreviations: $\mathrm{LSC}=$ large single copy region, $\mathrm{SSC}=$ small single copy region, $\mathrm{IRA} / \mathrm{B}=$ inverted repeat A/B. Fig. S2. Chloroplast genome of Cuscuta campestris. Transcription is counterclockwise around the outside of the circle and clockwise inside. The grey inner graph is GC content. Abbreviations: $\mathrm{LSC}=$ large single copy region, $\mathrm{SSC}=$ small single copy region, IRA/B = inverted repeat $A / B$. Fig. S3. Dispersed repeats in the mitogenomes of Cuscuta australis (left) and C. campestris (right). Generated with Circoletto (http://tools.bat.infspire.org/circoletto/). Fig. S4. Read depth for mitogenome assemblies of Cuscuta australis (top) and C. campestris (bottom). Short read depth is in black while long read depth is in green. Breakpoints between contigs in C. australis are blue lines. Minimum and mean read depths are indicated, with colours of text matching respective read depth. Fig. S5. Edited sites in ccmFC from Cuscuta australis based on mapped transcriptomic reads and predicted by PREP-MT. Exons are indicated above the mapped reads, with circles corresponding to panels above showing the mismatches in more detail. Positions along the alignment are numbered, with positions not predicted by PREP-MT circled in blue, and those predicted but not observed by mapping circled in red. The table shows details of the results from PREP-MT. Table S1. Repeats recombination analysis for Cuscuta australis. The analysis is based on non- 
overlapping repeats $>500$ bp and $>90 \%$ identity (blastn evalue 1e-10), and mapping long reads to those repeats. Table S2. Repeats recombination analysis for Cuscuta campestris. The analysis is based on nonoverlapping repeats $>500 \mathrm{bp}$ and $>90 \%$ identity (blastn evalue 1e-10), and mapping long reads to those repeats.

Additional file 2: Fig. S6. Schematic of the structure of nad 1 in Cuscuta australis based on DNA alignment with C. campestris and Ipomoea nil and alignment of transcriptomic reads. At top, a Mauve alignment of the section from exon4 to exon5 (containing matR) is shown for the three species (from top to bottom: Ipomoea nil, Cuscuta australis, C. campestris). Direction of transcription is shown with arrows. Exon structure is shown below with mRNA reads aligned to the DNA sequences separated by 100 Ns. Black lines demarcate the exonic sequences. Coloured lines in aligned reads indicate mismatches, corresponding to putative editing sites in most cases. Figs. S7 and S8. BLASTN hits for reference mitogenomes and plastomes plotted along the mitogenomes of Cuscuta australis and C. campestris. Plastome hits are shown in green along the linear representations of the mitogenomes, while hits to reference mitogenomes are shown by order above. The percentage identity of the mitogenomic hits is coloured based on the legend at the right. Solanales is shaded, as the order to which Cuscuta belongs. Hits $>500$ bp in regions not hit by Solanales are circled in red as possible HGT candidates. Fig. S9. Phylogenetic trees of plastid hits in mitogenomes of Cuscuta australis and C. campestris, analysed with top BLASTN hits from NCBI. All support values from 1000 rapid bootstrap replicates are shown above branches. Hits correspond to Table 2 and include the location within each mitogenome. Cuscuta sequences from plastomes are highlighted in green, while Cuscuta mitogenomic sequences are highlighted in yellow. Scale bars indicate inferred nucleotide substitutions per site. Figs. S10, S11, S12 and S13. Phylogenetic trees for nuclear candidate copies of mitochondrial genes in Cuscuta australis and C. campestris in relation to known mitochondrial copies in other plants and top BLASTN hits from NCBI. Bootstrap support values from 1000 rapid bootstrap replicates are shown above branches. The name of each candidate gene copy is in bold adjacent to its respective tree. Mitogenomic reference sequences (and BLASTN hits to mitogenomes) are indicated. Sequences from Cuscuta are highlighted in blue. Scale bars indicate inferred nucleotide substitutions per site.

Additional file 3: Fig. S14. Phylogenetic trees for mitochondrial genes across angiosperms, with particular focus (red) on Cuscuta species. Bootstrap support $>60 \%$ is shown adjacent to nodes. Scale bars indicate inferred nucleotide substitutions per site. Groups are coloured according to the legend below, with the exception of Cuscuta (red). The names of the genes are indicated above their respective trees.

Additional file 4: Table S3. Reference plastomes used for annotation and BLASTN searches and their GenBank accession numbers. Table S4. Reference mitogenomes used for annotation, BLASTN searches and phylogenetic analysis and their GenBank accession numbers.

\section{Acknowledgements}

Not applicable.

\section{Authors' contributions}

BMA and GP conceived and designed the study. KK provided access to genomic data. BMA conducted bioinformatic assembly and analysis. BMA drafted the manuscript. All authors contributed to critically revising the manuscript. All authors read and approved the final version.

\section{Funding}

B. Anderson and G. Petersen were supported by the Department of Ecology, Environment and Plant Sciences, Stockholm University. Some assemblies and analyses were performed on computing resources provided by the Swedish National Infrastructure for Computing (SNIC) through Uppsala Multidisciplinary Center for Advanced Computational Science (UPPMAX) under Project SNIC 2018/8-336. K. Krause was supported by a grant from the Tromsø Research Foundation [grant number 16-TF-KK]. The funding bodies played no role in the design of the study; the collection, analysis, and interpretation of data; and the writing of the manuscript. Open Access funding provided by Stockholm University.

\section{Availability of data and materials}

Organelle genomes are deposited in GenBank under accessions BK059221 and BK059222 for the C. australis and C. campestris plastomes, and BK059197-BK059204 and BK016277 for the C. australis and C. campestris mitogenomes, respectively.

Accession numbers for reference sequences downloaded from Genbank are provided in Additional file 4. Sequence alignments used in phylogenetic analysis are included as supplementary material for an earlier version of this paper at https://doi.org/10.1101/2021.04.15.439983.

\section{Declarations}

Ethics approval and consent to participate

Not applicable.

\section{Consent for publication}

Not applicable.

\section{Competing interests}

The authors declare that they have no competing interests.

\section{Author details}

${ }^{1}$ Department of Ecology, Environment and Plant Sciences, Stockholm University, SE-106 91 Stockholm, Sweden. ${ }^{2}$ Department of Arctic and Marine Biology, UiT The Arctic University of Norway, Breivika, 9037 Tromsø, Norway.

Received: 21 September 2020 Accepted: 19 October 2021

Published online: 12 November 2021

\section{References}

1. Koonin EV, Makarova KS, Aravind L. Horizontal gene transfer in prokaryotes: quantification and classification. Annu Rev Microbiol. 2001;55:709-42.

2. Keeling PJ, Palmer JD. Horizontal gene transfer in eukaryotic evolution. Nat Rev Genet. 2008;9:605-18.

3. Dunning LT, Olofsson JK, Parisod C, Choudhury RR, Moreno-Villena JJ, Yang $Y$, et al. Lateral transfers of large DNA fragments spread functional genes among grasses. Proc Natl Acad Sci U S A. 2019;116:4416-25.

4. Yang Z, Wafula EK, Kim G, Shahid S, McNeal JR, Ralph PE, et al. Convergent horizontal gene transfer and cross-talk of mobile nucleic acids in parasitic plants. Nat Plants. 2019. https://doi.org/10.1038/s41477-019-0458-0.

5. Stegemann S, Bock R. Exchange of genetic material between cells in plant tissue grafts. Science. 2009;324:649-51.

6. Kim G, LeBlanc ML, Wafula EK, de Pamphilis CW, Westwood JH. Genomicscale exchange of mRNA between a parasitic plant and its hosts. Science. 2014;345:808-11.

7. Rice DW, Alverson AJ, Richardson AO, Young GJ, Sanchez-Puerta MV Munzinger J, et al. Horizontal transfer of entire genomes via mitochondrial fusion in the angiosperm Amborella. Science. 2013:342:1468-73.

8. Richardson AO, Palmer JD. Horizontal gene transfer in plants. J Exp Bot. 2007:58:1-9.

9. Bergthorsson U, Adams KL, Thomason B, Palmer JD. Widespread horizontal transfer of mitochondrial genes in flowering plants. Nature. 2003;424:197201.

10. Won H, Renner SS. Horizontal gene transfer from flowering plants to Gnetum. Proc Natl Acad Sci U S A. 2003:100:10824-9.

11. Koulintchenko M, Konstantinov $Y$, Dietrich A. Plant mitochondria actively import DNA via the permeability transition pore complex. EMBO J. 2003;22: 1245-54.

12. Logan DC. Plant mitochondrial dynamics. Biochim Biophys Acta. 1763;2006: 430-41.

13. Arimura S-I, Yamamoto J, Aida GP, Nakazono M, Tsutsumi N. Frequent fusion and fission of plant mitochondria with unequal nucleoid distribution. Proc Natl Acad Sci U S A. 2004;101:7805-8.

14. Davis CC, Xi Z. Horizontal gene transfer in parasitic plants. Curr Opin Plant Biol. 2015;26:14-9.

15. Petersen G, Anderson B, Braun H-P, Meyer EH, Møller IM. Mitochondria in parasitic plants. Mitochondrion. 2020;52:173-82.

16. Wicke S, Naumann J. Molecular Evolution of Plastid Genomes in Parasitic Flowering Plants. In: Chaw S-M, Jansen RK, editors. Advances in Botanical Research: Academic Press; 2018. p. 315-47. https://doi.org/10.1016/bs.abr.2 017.11.014. 
17. Krause K. From chloroplasts to "cryptic" plastids: evolution of plastid genomes in parasitic plants. Curr Genet. 2008;54:111.

18. Petersen G, Cuenca A, Møller IM, Seberg O. Massive gene loss in mistletoe (Viscum, Viscaceae) mitochondria. Sci Rep. 2015;5:17588.

19. Skippington E, Barkman TJ, Rice DW, Palmer JD. Miniaturized mitogenome of the parasitic plant Viscum scurruloideum is extremely divergent and dynamic and has lost all nad genes. Proc Natl Acad Sci U S A. 2015;112: E3515-24.

20. Zervas A, Petersen G, Seberg O. Mitochondrial genome evolution in parasitic plants. BMC Evol Biol. 2019;19:87

21. Fan W, Zhu A, Kozaczek M, Shah N, Pabón-Mora N, González F, et al. Limited mitogenomic degradation in response to a parasitic lifestyle in Orobanchaceae. Sci Rep. 2016;6:36285.

22. Sanchez-Puerta MV, Edera A, Gandini CL, Williams AV, Howell KA, Nevill PG, et al. Genome-scale transfer of mitochondrial DNA from legume hosts to the holoparasite Lophophytum mirabile (Balanophoraceae). Mol Phylogenet Evol. 2019;132:243-50.

23. Sanchez-Puerta M, García LE, Wohlfeiler J, Ceriotti LF. Unparalleled replacement of native mitochondrial genes by foreign homologs in a holoparasitic plant. New Phytol. 2017;214:376-87.

24. Xi Z, Wang Y, Bradley RK, Sugumaran M, Marx CJ, Rest JS, et al. Massive mitochondrial gene transfer in a parasitic flowering plant clade. PLoS Genet. 2013;9:e1003265.

25. Garcia LE, Edera AA, Palmer JD, Sato H, Sanchez-Puerta MV. Horizontal gene transfers dominate the functional mitochondrial gene space of a holoparasitic plant. New Phytol. 2020. https://doi.org/10.1111/nph.16926.

26. Dawson JH, Musselman LJ, Wolswinkel P, Dörr I. Biology and control of Cuscuta. Rev Weed Sci. 1994;6:265-317.

27. van der Kooij TA, Krause K, Dörr I, Krupinska K. Molecular, functional and ultrastructural characterisation of plastids from six species of the parasitic flowering plant genus Cuscuta. Planta. 2000;210:701-7.

28. McNeal JR, Kuehl JV, Boore JL, de Pamphilis CW. Complete plastid genome sequences suggest strong selection for retention of photosynthetic genes in the parasitic plant genus Cuscuta. BMC Plant Biol. 2007;7:57.

29. Funk HT, Berg S, Krupinska K, Maier UG, Krause K. Complete DNA sequences of the plastid genomes of two parasitic flowering plant species, Cuscuta reflexa and Cuscuta gronovii. BMC Plant Biol. 2007;7:45.

30. Stefanović S, Olmstead RG. Down the slippery slope: plastid genome evolution in Convolvulaceae. J Mol Evol. 2005;61:292-305.

31. Revill MJW, Stanley S, Hibberd JM. Plastid genome structure and loss of photosynthetic ability in the parasitic genus Cuscuta. J Exp Bot. 2005;56: 2477-86.

32. Birschwilks M, Haupt S, Hofius D, Neumann S. Transfer of phloem-mobile substances from the host plants to the holoparasite Cuscuta sp. J Exp Bot. 2006;57:911-21.

33. Vogel A, Schwacke R, Denton AK, Usadel B, Hollmann J, Fischer K, et al. Footprints of parasitism in the genome of the parasitic flowering plant Cuscuta campestris. Nat Commun. 2018;9:2515.

34. Mower JP, Stefanović S, Young GJ, Palmer JD. Plant genetics: gene transfer from parasitic to host plants. Nature. 2004;432:165-6.

35. Mower JP, Stefanović S, Hao W, Gummow JS, Jain K, Ahmed D, et al. Horizontal acquisition of multiple mitochondrial genes from a parasitic plant followed by gene conversion with host mitochondrial genes. BMC Biol. 2010;8:150.

36. Park S, Grewe F, Zhu A, Ruhlman TA, Sabir J, Mower JP, et al. Dynamic evolution of Geranium mitochondrial genomes through multiple horizontal and intracellular gene transfers. New Phytol. 2015;208:570-83.

37. Jiang L, Wijeratne AJ, Wijeratne S, Fraga M, Meulia T, Doohan D, et al. Profiling mRNAs of two Cuscuta species reveals possible candidate transcripts shared by parasitic plants. PLoS One. 2013;8:e81389.

38. Zhang $Y$, Fernandez-Aparicio M, Wafula EK, Das M, Jiao Y, Wickett NJ, et al. Evolution of a horizontally acquired legume gene, albumin 1, in the parasitic plant Phelipanche aegyptiaca and related species. BMC Evol Biol. 2013;13:48.

39. Zhang D, Qi J, Yue J, Huang J, Sun T, Li S, et al. Root parasitic plant Orobanche aegyptiaca and shoot parasitic plant Cuscuta australis obtained Brassicaceae-specific strictosidine synthase-like genes by horizontal gene transfer. BMC Plant Biol. 2014;14:19.

40. Zhang $Y$, Wang D, Wang Y, Dong H, Yuan Y, Yang W, et al. Parasitic plant dodder (Cuscuta spp.): a new natural Agrobacterium-to-plant horizontal gene transfer species. Sci China Life Sci. 2020;63:312-6.
41. Sun G, Xu Y, Liu H, Sun T, Zhang J, Hettenhausen C, et al. Large-scale gene losses underlie the genome evolution of parasitic plant Cuscuta australis. Nat Commun. 2018:9:2683.

42. Krause K, Dieckmann CL. Analysis of transcription asymmetries along the tRNAE-COB operon: evidence for transcription attenuation and rapid RNA degradation between coding sequences. Nucleic Acids Res. 2004:32:6276-83.

43. Small I, Peeters N, Legeai F, Lurin C. Predotar: a tool for rapidly screening proteomes for $\mathrm{N}$-terminal targeting sequences. Proteomics. 2004;4:1581-90.

44. Adams KL, Qiu Y-L, Stoutemyer M, Palmer JD. Punctuated evolution of mitochondrial gene content: high and variable rates of mitochondrial gene loss and transfer to the nucleus during angiosperm evolution. Proc Natl Acad Sci U S A. 2002;99:9905-12.

45. Kubo N, Arimura S-I. Discovery of the rp/10 gene in diverse plant mitochondrial genomes and its probable replacement by the nuclear gene for chloroplast RPL10 in two lineages of angiosperms. DNA Res. 2010;17:1-9.

46. Adams KL, Palmer JD. Evolution of mitochondrial gene content: gene loss and transfer to the nucleus. Mol Phylogenet Evol. 2003;29:380-95.

47. Wolfe KH, Li WH, Sharp PM. Rates of nucleotide substitution vary greatly among plant mitochondrial, chloroplast, and nuclear DNAs. Proc Natl Acad Sci U S A. 1987;84:9054-8.

48. Laroche J, Li P, Maggia L, Bousquet J. Molecular evolution of angiosperm mitochondrial introns and exons. Proc Natl Acad Sci U S A. 1997;94:5722-7.

49. Sloan DB, Alverson AJ, Chuckalovcak JP, Wu M, McCauley DE, Palmer JD, et al. Rapid evolution of enormous, multichromosomal genomes in flowering plant mitochondria with exceptionally high mutation rates. PLoS Biol. 2012;10:e1001241.

50. Adams KL, Ong HC, Palmer JD. Mitochondrial gene transfer in pieces: fission of the ribosomal protein gene rp/2 and partial or complete gene transfer to the nucleus. Mol Biol Evol. 2001;18:2289-97.

51. Liu S-L, Zhuang Y, Zhang P, Adams KL. Comparative analysis of structural diversity and sequence evolution in plant mitochondrial genes transferred to the nucleus. Mol Biol Evol. 2009;26:875-91.

52. Petersen G, Cuenca A, Zervas A, Ross GT, Graham SW, Barrett CF, et al. Mitochondrial genome evolution in Alismatales: size reduction and extensive loss of ribosomal protein genes. PLoS One. 2017;12:e0177606.

53. Mower JP, Bonen L. Ribosomal protein L10 is encoded in the mitochondrial genome of many land plants and green algae. BMC Evol Biol. 2009;9:265.

54. Gandini CL, Sanchez-Puerta MV. Foreign plastid sequences in plant mitochondria are frequently acquired via mitochondrion-to-mitochondrion horizontal transfer. Sci Rep. 2017;7:43402.

55. Kozik A, Rowan BA, Lavelle D, Berke L, Schranz ME, Michelmore RW, et al. The alternative reality of plant mitochondrial DNA: one ring does not rule them all. PLoS Genet. 2019;15:e1008373.

56. Mower JP, Case AL, Floro ER, Willis JH. Evidence against equimolarity of large repeat arrangements and a predominant master circle structure of the mitochondrial genome from a monkeyflower (Mimulus guttatus) lineage with cryptic CMS. Genome Biol Evol. 2012;4:670-86.

57. Mower JP, Sloan DB, Alverson AJ. Plant mitochondrial genome diversity: the genomics revolution. In: Wendel JF, Greilhuber J, Dolezel J, Leitch IJ, editors. Plant genome diversity volume 1: plant genomes, their residents, and their evolutionary dynamics. Vienna: Springer Vienna; 2012. p. 123-44.

58. Marienfeld J, Unseld M, Brennicke A. The mitochondrial genome of Arabidopsis is composed of both native and immigrant information. Trends Plant Sci. 1999:4:495-502.

59. de Longevialle AF, Small ID, Lurin C. Nuclearly encoded splicing factors implicated in RNA splicing in higher plant organelles. Mol Plant. 2010;3:691-705.

60. Bonen L. Cis- and trans-splicing of group II introns in plant mitochondria. Mitochondrion. 2008;8:26-34

61. Kim S, Yoon M-K. Comparison of mitochondrial and chloroplast genome segments from three onion (Allium cepa L.) cytoplasm types and identification of a trans-splicing intron of cox2. Curr Genet. 2010;56:177-88.

62. Glanz S, Kück U. Trans-splicing of organelle introns - a detour to continuous RNAs. Bioessays. 2009;31:921-34.

63. des Francs-Small CC, Kroeger T, Zmudjak M, Ostersetzer-Biran O, Rahimi N Small I, et al. A PORR domain protein required for rp/2 and $\mathrm{ccmF}_{C}$ intron splicing and for the biogenesis of c-type cytochromes in Arabidopsis mitochondria: PORR domain mitochondrial splicing factor. Plant J. 2012;69: 996-1005.

64. Giegé $P$, Rayapuram N, Meyer EH, Grienenberger JM, Bonnard G. CcmF involved in cytochrome $c$ maturation is present in a large sized complex in wheat mitochondria. FEBS Lett. 2004;563:165-9. 
65. Choi I-S, Ruhlman TA, Jansen RK. Comparative mitogenome analysis of the genus Trifolium reveals independent gene fission of $\mathrm{cmFn}$ and intracellular gene transfers in Fabaceae. Int J Mol Sci. 2020;21:1959.

66. Andrews S. FastQC. Babraham bioinformatics. 2018. https://www.bioinforma tics.babraham.ac.uk/projects/fastqc/. Accessed Feb 2019.

67. Bushnell B. BBMap: a fast, accurate, splice-aware aligner. Lawrence Berkeley National Laboratory; 2014. https://sourceforge.net/projects/bbmap/. Accessed 2 Oct 2018.

68. Koren S, Walenz BP, Berlin K, Miller JR, Bergman NH, Phillippy AM. Canu: scalable and accurate long-read assembly via adaptive k-mer weighting and repeat separation. Genome Res. 2017;27:722-36

69. Carpenter EJ, Matasci N, Ayyampalayam S, Wu S, Sun J, Yu J, et al. Access to RNA-sequencing data from 1,173 plant species: the 1000 plant transcriptomes initiative (1KP). Gigascience. 2019;8. https://doi.org/10.1093/ gigascience/giz126.

70. Wei C, Tao X, Li M, He B, Yan L, Tan X, et al. De novo transcriptome assembly of Ipomoea nil using Illumina sequencing for gene discovery and SSR marker identification. Mol Gen Genomics. 2015;290:1873-84.

71. Hoshino A, Jayakumar V, Nitasaka E, Toyoda A, Noguchi H, Itoh T, et al. Genome sequence and analysis of the Japanese morning glory Ipomoea nil. Nat Commun. 2016:7:13295.

72. Altschul SF, Gish W, Miller W, Myers EW, Lipman DJ. Basic local alignment search tool. J Mol Biol. 1990:215:403-10.

73. Altschul SF, Madden TL, Schäffer AA, Zhang J, Zhang Z, Miller W, et al. Gapped BLAST and PSI-BLAST: a new generation of protein database search programs. Nucleic Acids Res. 1997;25:3389-402.

74. Camacho C, Coulouris G, Avagyan V, Ma N, Papadopoulos J, Bealer K, et al. BLAST+: architecture and applications. BMC Bioinformatics. 2009:10:421.

75. Mapleson D, Garcia Accinelli G, Kettleborough G, Wright J, Clavijo BJ. KAT: a K-mer analysis toolkit to quality control NGS datasets and genome assemblies. Bioinformatics. 2017;33:574-6.

76. Wick RR, Judd LM, Gorrie CL, Holt KE. Unicycler: resolving bacterial genome assemblies from short and long sequencing reads. PLoS Comput Biol. 2017; 13:e1005595.

77. Bankevich A, Nurk S, Antipov D, Gurevich AA, Dvorkin M, Kulikov AS, et al. SPAdes: a new genome assembly algorithm and its applications to singlecell sequencing. J Comput Biol. 2012;19:455-77.

78. Wick RR, Schultz MB, Zobel J, Holt KE. Bandage: interactive visualization of de novo genome assemblies. Bioinformatics. 2015;31:3350-2.

79. Li H. Minimap and miniasm: fast mapping and de novo assembly for noisy long sequences. Bioinformatics. 2016;32:2103-10.

80. Vaser R, Sović I, Nagarajan N, Šikić M. Fast and accurate de novo genome assembly from long uncorrected reads. Genome Res. 2017;27:737-46.

81. Dierckxsens N, Mardulyn P, Smits G. NOVOPlasty: de novo assembly of organelle genomes from whole genome data. Nucleic Acids Res. 2017:45:e18.

82. Li H, Handsaker B, Wysoker A, Fennell T, Ruan J, Homer N, et al. The sequence alignment/map format and SAMtools. Bioinformatics. 2009;25: 2078-9.

83. Katoh K, Standley DM. MAFFT multiple sequence alignment software version 7: improvements in performance and usability. Mol Biol Evol. 2013; 30:772-80.

84. Robinson JT, Thorvaldsdóttir H, Winckler W, Guttman M, Lander ES, Getz G, et al. Integrative genomics viewer. Nat Biotechnol. 2011;29:24-6.

85. Alverson AJ, Wei X, Rice DW, Stern DB, Barry K, Palmer JD. Insights into the evolution of mitochondrial genome size from complete sequences of Citrullus lanatus and Cucurbita pepo (Cucurbitaceae). Mol Biol Evol. 2010;27: 1436-48.

86. Eddy SR. Accelerated profile HMM searches. PLoS Comput Biol. 2011;7: e1002195.

87. Lowe TM, Eddy SR. tRNAscan-SE: a program for improved detection of transfer RNA genes in genomic sequence. Nucleic Acids Res. 1997;25:955-64.

88. Darzentas N. Circoletto: visualizing sequence similarity with Circos Bioinformatics. 2010;26:2620-1.

89. Benson G. Tandem repeats finder: a program to analyze DNA sequences. Nucleic Acids Res. 1999;27:573-80.

90. Greiner S, Lehwark P, Bock R. OrganellarGenomeDRAW (OGDRAW) version 1. 3.1: expanded toolkit for the graphical visualization of organellar genomes. Nucleic Acids Res. 2019;47:W59-64.

91. Waterhouse AM, Procter JB, Martin DMA, Clamp M, Barton GJ. Jalview version 2 - a multiple sequence alignment editor and analysis workbench. Bioinformatics. 2009;25:1189-91.
92. Zuker M. Mfold web server for nucleic acid folding and hybridization prediction. Nucleic Acids Res. 2003:31:3406-15.

93. Mower JP. PREP-Mt: predictive RNA editor for plant mitochondrial genes. BMC Bioinformatics. 2005;6:96.

94. Darling ACE, Mau B, Blattner FR, Perna NT. Mauve: multiple alignment of conserved genomic sequence with rearrangements. Genome Res. 2004;14 1394-403.

95. Stamatakis A. RAxML version 8: a tool for phylogenetic analysis and postanalysis of large phylogenies. Bioinformatics. 2014;30:1312-3.

96. Sievers F, Wilm A, Dineen D, Gibson TJ, Karplus K, Li W, et al. Fast, scalable generation of high-quality protein multiple sequence alignments using Clustal omega. Mol Syst Biol. 2011;7 https://www.embopress.org/doi/abs/1 $0.1038 / \mathrm{msb} .2011 .75$

97. Suyama M, Torrents D, Bork P. PAL2NAL: robust conversion of protein sequence alignments into the corresponding codon alignments. Nucleic Acids Res. 2006;34(Web Server issue):W609-12.

98. Sanchez-Puerta MV, Abbona CC, Zhuo S, Tepe EJ, Bohs L, Olmstead RG, et al. Multiple recent horizontal transfers of the cox1 intron in Solanaceae and extended co-conversion of flanking exons. BMC Evol Biol. 2011;11:277.

99. Paradis E, Schliep K. Ape 5.0: an environment for modern phylogenetics and evolutionary analyses in R. Bioinformatics. 2019;35:526-8.

100. Phanstiel DH, Boyle AP, Araya CL, Snyder MP. Sushi.R: flexible, quantitative and integrative genomic visualizations for publication-quality multi-panel figures. Bioinformatics. 2014;30:2808-10.

101. Sawyer S. Statistical tests for detecting gene conversion. Mol Biol Evol. 1989; $6.526-38$

\section{Publisher's Note}

Springer Nature remains neutral with regard to jurisdictional claims in published maps and institutional affiliations.

Ready to submit your research? Choose BMC and benefit from:

- fast, convenient online submission

- thorough peer review by experienced researchers in your field

- rapid publication on acceptance

- support for research data, including large and complex data types

- gold Open Access which fosters wider collaboration and increased citations

- maximum visibility for your research: over $100 \mathrm{M}$ website views per year

At BMC, research is always in progress.

Learn more biomedcentral.com/submissions 